\title{
Quelle dimension argumentative dans les carnets de recherche en sciences humaines?
}

The argumentative dimension of academic blogs in the Humanities

\section{Ingrid Mayeur}

\section{(2) OpenEdition}

1 Journals

\section{Édition électronique}

URL : http://journals.openedition.org/aad/2535

DOI : $10.4000 /$ aad. 2535

ISSN : 1565-8961

Éditeur

Université de Tel-Aviv

\section{Référence électronique}

Ingrid Mayeur, «Quelle dimension argumentative dans les carnets de recherche en sciences humaines ? ", Argumentation et Analyse du Discours [En ligne], 20 | 2018, mis en ligne le 15 avril 2018, consulté le 23 septembre 2019. URL : http://journals.openedition.org/aad/2535; DOI : 10.4000/aad. 2535

\section{Ce document a été généré automatiquement le 23 septembre 2019}

\section{c) (7) $(9)$}

Argumentation \& analyse du discours est mis à disposition selon les termes de la licence Creative Commons Attribution - Pas d'Utilisation Commerciale - Pas de Modification 4.0 International. 


\section{Quelle dimension argumentative dans les carnets de recherche en sciences humaines?}

The argumentative dimension of academic blogs in the Humanities

Ingrid Mayeur

\section{NOTE DE L'AUTEUR}

Je tiens à remercier Marin Dacos et l'équipe d'OpenEdition pour leur disponibilité ainsi que l'accès à certains documents, qui ont aidé à la rédaction de cet article.

1 Les formes de communication de la recherche se trouvent affectées par les conditions particulières de production et de diffusion autorisées par les dispositifs numériques. Dans le domaine de l'écrit, les genres de l'article et de la monographie évoluent, notamment par l'ajout de métadonnées qui en permettent l'éditorialisation (Vitali Rosati 2016) au sein d'une plateforme de communication scientifique. S'y joignent des formats natifs du Web tel que le billet de blog, publié sur un carnet de chercheur ou un site collectif; ou des écrits que l'on rattache à la littérature grise (rapports, notes préliminaires, etc.) déposés au sein d'archives ouvertes prévues à cet effet.

2 Article et monographie s'adressent généralement à un lectorat de pairs dans une communauté disciplinaire définie (si l'on excepte les écrits de vulgarisation, qui occupent une place distincte dans le champ éditorial). Ils relèvent ainsi de la catégorie des discours fermés (Charaudeau et Maingueneau 2002: 261), établis par des spécialistes pour des spécialistes. Le numérique contribue à brouiller les frontières ; outre la possibilité technique, avec l'accès ouvert, de toucher un public extraacadémique, l'open Science ${ }^{1}$ postule une nouvelle temporalité de la recherche préconisant les échanges immédiats dans une communauté scientifique globalisée. L' Open Science encourage ainsi un double mouvement de médiatisation et de médiation de la recherche, dans le but de répondre à une demande émanant du grand public 
(Commission Européenne 2016 : 35). Si les études linguistiques des écrits scientifiques, s'appuyant au besoin sur la notion de dimension argumentative (Amossy 2000), ont insisté sur le dialogisme (Volochinov 2010) qui traverse le discours savant (chaque énoncé se positionnant par rapport aux énoncés antérieurs, voire à venir) et les conditions particulières d'énonciation qui visent à le légitimer, elles se préoccupent surtout d'un discours adressé à un public homogène constitué d'acteurs du monde universitaire, soit un discours fermé.

3 La présente contribution entend questionner la dimension argumentative d'écrits scientifiques natifs du Web que sont les billets de blog, à partir d'une étude de cas tirés de la plateforme Hypothèses. Cet espace de blogging scientifique hébergé sur la plateforme OpenEdition, lancé officiellement en 2010, compte actuellement à son catalogue près de 2500 carnets de recherche en sciences humaines et sociales ${ }^{2}$. L'auditoire hétérogène que présuppose le contexte de l'open Science amène à s'interroger sur les stratégies rhétoriques d'adaptation au public mises en œuvre par les carnetiers pour favoriser l'appropriation des savoirs diffusés. L'étude de la dimension argumentative des écrits de blogs, qui intègre dans l'analyse du discours scientifique la préoccupation d'une action sur l'auditoire, fournit des outils pour expliquer le recours aux moyens de preuves rhétoriques au sein de textes qui ne possèdent pas une visée argumentative explicite. Ce sont ces outils dont je voudrais tester ici l'opérativité.

\section{La dimension argumentative du discours scientifique}

4 Pour cerner la notion de discours scientifique, on recourra à la définition proposée par Rinck qui en fait un « discours produit dans le cadre de l'activité de recherche à des fins de construction et de diffusion du savoir » (2010: \$2). Dans l'imaginaire courant, on tend à considérer le texte scientifique comme empreint d'objectivité; celle-ci se traduirait au plan discursif par l'effacement énonciatif du locuteur et la neutralité axiologique du lexique. La tendance à l'objectivité garantirait en quelque sorte la validité de la recherche, puisque l'auteur n'aurait plus qu'à s'effacer devant l'évidence empirique des résultats. Or, cette représentation s'est vue mise en cause par les approches rhétoriques et linguistiques des textes scientifiques, qui ont relevé depuis les années 1960 (Boch et Rinck 2010) que le discours scientifique était traversé de bout en bout par le recours aux moyens de persuasion. De ce point de vue, l'effacement énonciatif dont témoignent les textes dits " théoriques » (dans la typologie énonciative des textes de Bronckart et al. 1994 [1985] : 83) peut être perçu comme une stratégie rhétorique.

5 Une livraison de la revue Lidil intitulée «Énonciation et rhétorique dans l'écrit scientifique » (Boch et Rinck éds. 2010) étudie précisément la manière dont les locuteurs des écrits scientifiques construisent leur positionnement énonciatif et manifestent de ce fait l'appropriation discursive d'un habitus académique et disciplinaire. L'ouvrage dirigé par Tutin et Grossmann présentant les résultats du projet Scientext (2013) porte également une attention particulière à la question de l'auctorialité, et participe du même mouvement de remise en cause de la neutralité des écrits scientifiques ${ }^{3}$. À la suite de travaux récents que corroborent leurs propres observations sur le corpus de Scientext, les auteurs signalent, au sein des textes 
scientifiques étudiés, « une importante présence de l'auteur en sciences humaines et en sciences sociales» (2013: 13). Ils affirment ainsi que "l'écrit scientifique est véritablement un texte argumentatif où la dimension rhétorique est fortement présente " (ibid.) Si, en effet, le discours scientifique s'attache à construire un savoir à partir d'hypothèses validées, il s'agit rarement de convaincre explicitement le lecteur de suivre une proposition $\mathrm{A}$ au détriment d'une proposition $\mathrm{B}$.

Les travaux d'Amossy sur l'argumentation dans le discours permettent de distinguer entre visée argumentative - où l'argumentation portant sur un choix à faire entre deux positions antagonistes est explicite -, et dimension argumentative où le débat reste tacite (Amossy 2000 : 24). La notion de dimension argumentative est susceptible de servir l'étude des écrits scientifiques, dès lors que la démarche de recherche s'inscrit dans un horizon de questionnement plus large, par rapport aux positions d'autres auteurs. La prise en compte du dialogisme intrinsèque de tout énoncé s'accompagne d'une réévaluation du rôle argumentatif de l'ethos et du pathos, parfois sous-estimé dans les études centrées sur le logos (ibid.: 4). D'où une attention particulière portée à l'auditoire, notion issue de la rhétorique aristotélicienne et retravaillée par Perelman et Olbrechts-Tyteca qui en font une image mentale du public construite par l'orateur, en fonction de laquelle on construira l'argumentation (Perelman et Olbrecht-Tyteca 1988 [1958]).

\section{L'écriture en ligne : textes et discours numériques}

7 Un apport de Perelman et Olbrechts-Tyteca est d'élargir les préceptes issus la rhétorique aristotélicienne, orale, aux discours écrits. Or, les moyens de discourir et d'argumenter dans l'environnement numérique diffèrent sensiblement de ce qui a cours non seulement dans les échanges oraux mais également dans le domaine de l'imprimé. Chartier avait ainsi montré que le numérique entraînait une rupture de l'ordre des discours, sur laquelle repose la culture écrite occidentale, et par conséquent de l'ordre des raisons $(2006: 18)$ :

Du côté de l'auteur, la textualité électronique permet de développer des démonstrations selon une logique qui n'est plus nécessairement linéaire ou déductive [...]. Du côté du lecteur, la validation ou la récusation d'un argument peut désormais s'appuyer sur la consultation des textes [...] qui sont l'objet même de l'étude, à condition, évidemment, qu'ils soient accessibles en une forme numérisée [...]. Il y a là une mutation épistémologique fondamentale qui transforme profondément les techniques de la preuve et les modalités de construction et de validation des discours de savoir.

La matérialité du texte numérique transforme ainsi les conditions de production et de réception du discours savant, et en particulier ses modes de validation.

Dans le champ de l'analyse du discours, les travaux de Paveau sur les technologies discursives (Paveau 2017) ont bien saisi cette matérialité particulière du discours numérique, qui imbrique étroitement les composantes linguistiques et techniques. Les propriétés matérielles du discours en ligne posent la question de l'intégrité des données étudiées (Paveau 2012): délinéarisation du discours par les hypertextes, augmentation par l'ajout de commentaires, technogenres spécifiques au Web et plurisémioticité de l'énoncé intégrant images, vidéos ou animations. Ces discours anticipent des pratiques d'écrilectures (Paveau 2016) où la navigation hypertextuelle 
autorise des appropriations textuelles variées: le lecteur est amené à participer à l'élaboration du texte, par sa pratique de lecture et les manipulations qu'elle induit.

Ainsi qu'on le voit, l'usager se trouve activement sollicité par le discours numérique. Si la notion de dimension argumentative suppose la volonté d'agir sur l'allocutaire, cette action est ici très concrète et implique une manipulation physique de sa part (cliquer sur un hyperlien, activer les ascenseurs de défilement etc.) - c'est ainsi que Saemmer (2015) évoque l'anticipation des pratiques comme des «figures de la lecture», prises laissées à l'attention du lecteur pour une action sur le texte. Le discours scientifique numérique anticipera des usages précis selon l'image de l'auditoire construite par le locuteur.

\section{Le dispositif éditorial des écrits scientifiques numériques}

11 Le numérique ne reconfigure pas seulement l'ordre des discours; c'est également l'industrie de la publication des textes scientifiques qui se voit réorganisée par les possibilités de diffusion en réseau. Sans entrer ici dans le détail ${ }^{4}$, le numérique favorise l'émergence d'une communication scientifique en accès libre, qui ne fait pas forcément l'objet d'une révision préalable par les pairs (c'est le cas du blogging scientifique). Dans la sphère francophone, OpenEdition lance en 2010 la plateforme Hypothèses, qui postule, outre le public académique, un lectorat extra-universitaire : il y est ainsi spécifié que «[les textes] ne s'adressent pas exclusivement à des spécialistes, mais également au grand public $»^{5}$. Dacos et Mounier insistent d'ailleurs sur cet aspect de "brouillage éditorial » du carnet de recherche - le discours scientifique qui s'y inscrit ne peut dès lors plus être considéré comme «fermé » :

Par le blog, le chercheur s'adresse directement à un public qui n'est d'ailleurs plus segmenté [...]. Les différents billets, portant sur des sujets divers, rédigés de manières différentes s'adressent à des publics hétérogènes ou, mieux, ne préjugent ni de la qualité ni des compétences de ceux qui peuvent les lire (2010:5)

Le texte scientifique devient ainsi polychrésique, susceptible de soutenir différentes logiques sociales et de mener à des usages différents (Jeanneret 2014 :14). Seulement, la médiatisation d'un texte scientifique auprès d'un public hétérogène ne signifie pas grand-chose si elle ne vise pas l'appropriation des savoirs par ce public, en vue de leur circulation - processus que Jeanneret a mis en évidence par ses travaux sur la trivialité, définie comme « le caractère fondamental des processus qui permettent le partage, la transformation, l'appropriation des objets et des savoirs au sein d'un espace social hétérogène." (Jeanneret 2014: 20). De son point de vue, le discours constitue un "opérateur puissant de trivialité » (ibid.: 93) en ce qu'il met en lien, reformule, condense ou augmente les énoncés antérieurs. Les discours favorisent la circulation des savoirs à travers plusieurs pratiques, au rang desquelles figurent les stratégies rhétoriques d'adaptation au public. Jeanneret, comme sémioticien issu des études littéraires, ne fournit pas à proprement parler de méthodes pour les étudier, bien qu'il établisse un cadre théorique pertinent pour l'analyse critique des médias informatisés au sein desquels s'inscrivent ces discours. Les propositions théoriques d'Amossy fournissent les outils qui permettent de repérer les traces discursives de cet ajustement à l'auditoire. 
Il est difficile dans les limites de cet article de rendre compte de la richesse des appropriations du dispositif par les chercheurs en sciences humaines et sociale qui s'investissent dans un blog de recherche sur Hypothèses. C'est pourquoi je voudrais étudier plus précisément deux carnets, l'un donnant la parole à des chercheurs dans le contexte de la société française post-attentats, l'autre se rattachant à l'actualité de la recherche - en l'occurrence, celle d'une thèse en cours, relatée par deux historiens.

\section{4. Étude de cas}

\subsection{Choix du corpus et situation dans un interdiscours}

Pour mener à bien cette étude de cas, j'ai sélectionné deux carnets de recherche, UC@ctualité (section «Les attentats et après », corpus A) et ParenThèses (corpus B) dont j'ai extrait en tant qu'observables le texte de présentation ainsi que les cinq derniers billets en date du 10 mai 2017. On s'intéressera ici de manière privilégiée aux matériaux (techno)discursifs, mais il est évident qu'ils sont à mettre en relation avec l'économie globale du carnet de recherche (éléments visuels, énonciation éditoriale, structure et catégorisations) et, plus largement, à la logique éditoriale de la plateforme Hypothèses.

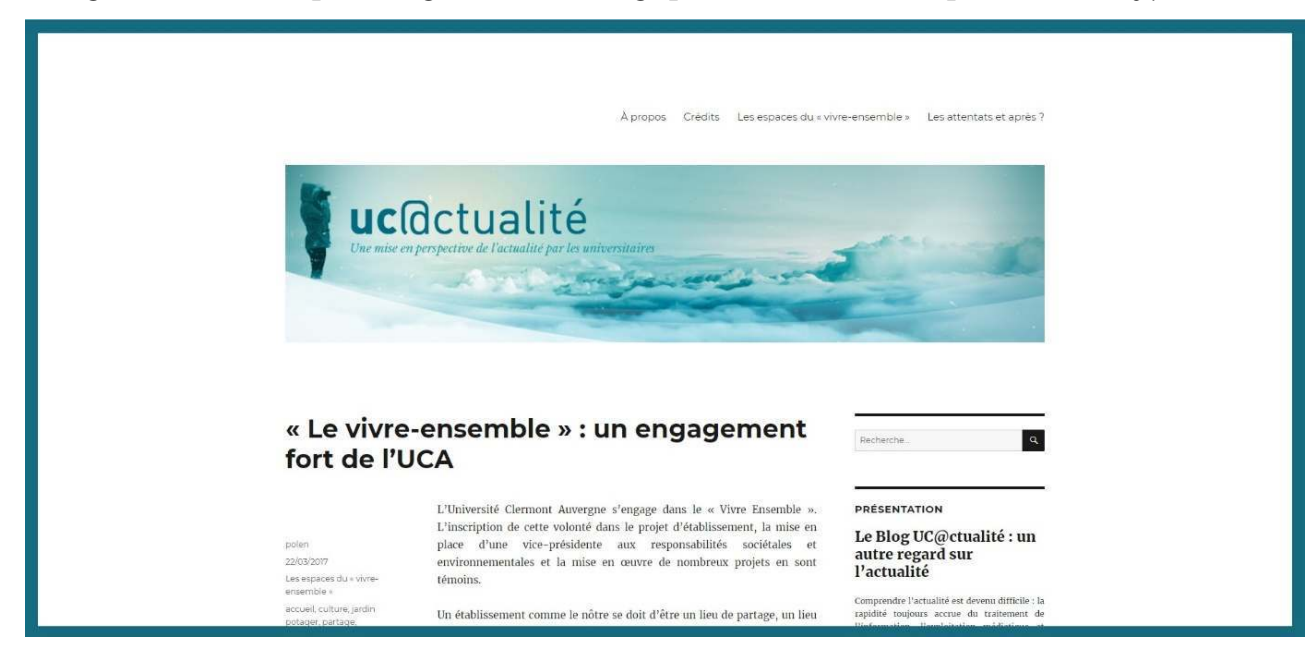

Fig. 1 : Bandeau du carnet UC@ctualité (capturé le 14 novembre 2017) 


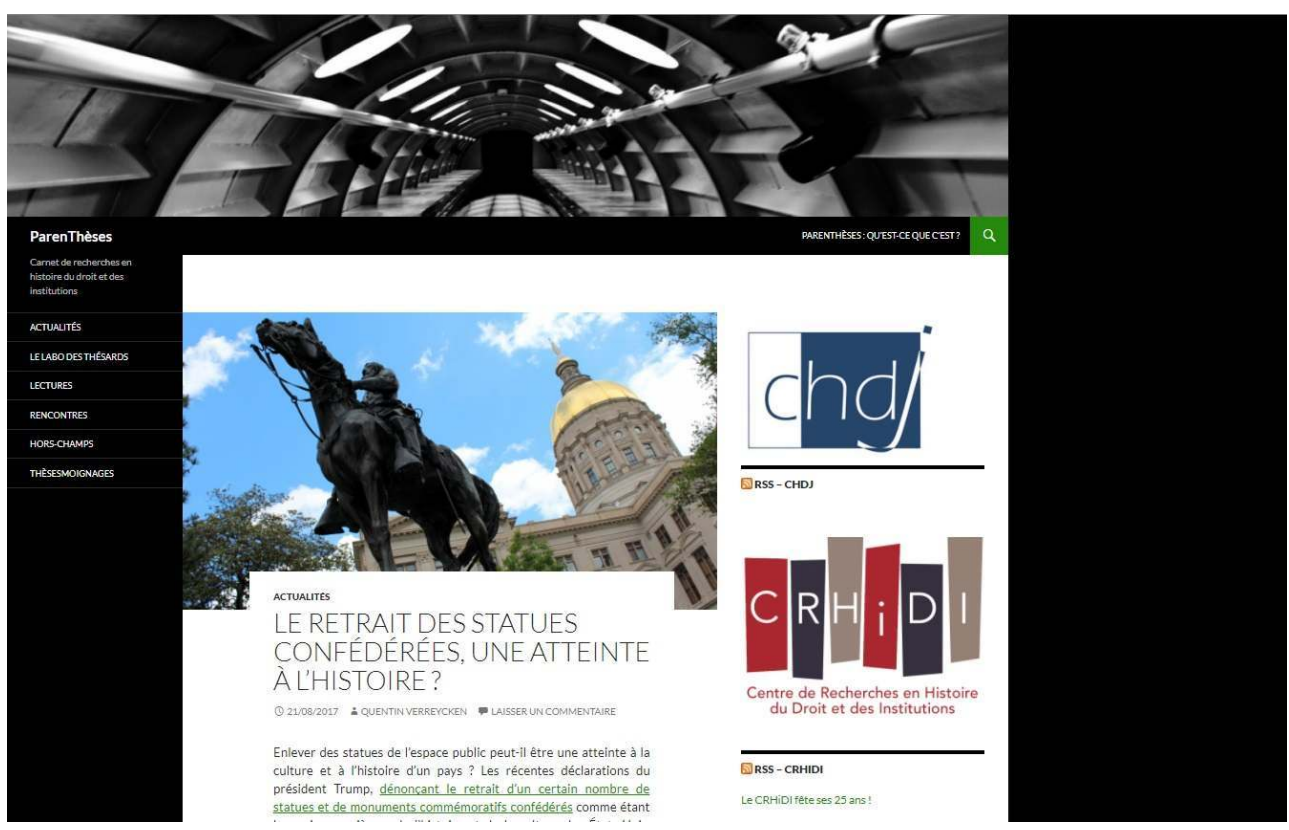

Fig. 2 : Bandeau du carnet ParenThèses (capturé le 23 novembre 2017)

Le premier carnet de recherche, UC@ctualité, suggère d'offrir comme mentionné par le sous-titre une « mise en perspective de l'actualité par les universitaires ». Si la rubrique «À propos " indique avant tout la volonté de fournir des matériaux à un lectorat de médiateurs (journalistes, enseignants etc.), l'éditorial intègre la possibilité d'un échange avec les collègues universitaires de toutes disciplines. Mais il est également question d'exploiter l'espace de liberté qu'offre le format du blog pour produire un savoir scientifique spécifique :

Ces contributions n'ont pas vertu à être des articles au sens de nos revues scientifiques de recherche. Il s'agit de laisser la place à la liberté intime de conviction de chaque auteur sans abandonner pour autant la rigueur scientifique et la distance nécessaires au traitement de l'événement. (corpus A, billet 1)

Le second, ParenThèses, est animé par deux doctorants en histoire :

Le carnet se veut, d'une part, un véritable «laboratoire méthodologie » [sic] des recherches doctorales de ses deux auteurs : seront publiés des billets portant sur des auteurs et des ouvrages qui nous sont chers, sur des réflexions théoriques et des paradigmes en usage dans notre discipline, de même que sur des hypothèses de travail que nous élaborons dans le cadre de nos thèses respectives. D'autre part, ParenThèses se veut également un lieu de promotion et de diffusion de l'histoire du droit et des institutions [...] Les auteurs de ce carnet tenteront, enfin, de s'aventurer en dehors de leur champ de recherche habituel pour se livrer à des réflexions sur les représentations du droit et des institutions dans les médias contemporains (corpus B, billet 1)

17 Il semble donc, selon les dires des auteurs à tout le moins, que les contenus des blogs UC@ctualité et ParenThèses correspondent à la définition donnée par Rinck du discours scientifique. Ils visent la production de savoirs d'un type autre que celui diffusé dans une revue disciplinaire à lectorat homogène, mais qui n'en reste pas moins pleinement intégré dans le domaine des écrits scientifiques. Ces savoirs se démarquent par leur inscription dans un interdiscours qui a vocation à dépasser les paradigmes d'un champ disciplinaire donné pour rejoindre une sphère d'activité sociale plus large au sein de laquelle l'activité scientifique se trouve intégrée. 
18 L'interdiscours au sein duquel s'exerce la prise de parole d'Uc@ctualité est celui d'une société française marquée par les attentats de 2015. Le carnet de recherche se présente comme une zone intermédiaire entre ce traitement de l'événement dans la temporalité immédiate « des médias et des réseaux sociaux » (corpus A, billet 1), et celui dont il fera l'objet dans un second temps par les "experts" dans des revues spécialisées, qui toutefois "ne permet pas toujours une vision pluridisciplinaire" (ibid.). Le carnet permet aussi l'« ouverture à la société » (que ce soit par les auteurs envisagés, pour l'essentiel des universitaires, mais également des artistes ou citoyens, ou par le lectorat attendu) considérée comme « vocation première » des scientifiques (ibid.).

Le carnet ParenThèses se rapproche pour une part de l'interdiscours classique du discours scientifique, où le chercheur se positionne au sein des paradigmes en cours dans sa discipline. Toutefois, pour une autre part, il s'agit de médiatiser la démarche de recherche dans son actualité, que ce soit par des réflexions sur des travaux en cours, des comptes rendus d'événements scientifiques ou des interviews de chercheurs. Le lien à l'actualité sociale n'est pas non plus absent : un billet comme «Le Moyen Âge vu par les politiques » (corpus B, billet 2) procède de l'actualité des débats parlementaires et questionne la pertinence des allusions à cette période historique par des hommes et femmes politiques non historiens, à travers le temps. À nouveau, le discours scientifique s'inscrit dans un interdiscours plus large où l'activité scientifique n'est plus en marge des autres activités sociales; ce qui a une incidence directe sur la construction de l'ethos du chercheur.

\subsection{L'ethos du chercheur-carnetier}

Le chercheur est amené, dans ses écrits, à construire discursivement son autorité afin de faire accepter de nouveaux savoirs à sa communauté disciplinaire. Sur quelles composantes discursives repose l'ethos du chercheur respectable ? D'après Amossy,

c'est donc le ton impersonnel et l'effacement énonciatif qui construisent une image fiable de l'homme de science. [...] Il se dit et se montre par le refus de s'exprimer et de se manifester. Ce faisant, il projette un ethos qui lui permet de proclamer son appartenance identitaire à la communauté à laquelle il entend s'intégrer, et par laquelle il demande à être reconnu (2010 : 191-192).

Ainsi qu'on l'a signalé sur la base de travaux antérieurs, l'effacement énonciatif n'est pas la marque d'une absence de subjectivité 6 . Au sein des carnets de recherches du corpus toutefois, cet effacement énonciatif n'a pas cours : au contraire, la majorité des billets étudiés sont écrits à la première personne, et bien souvent en «je »-pratique courante dans le blogging au demeurant. À l'origine, les blogs consistent soit en des pages personnelles recensant les nouveautés de la toile par des liens hypertextes, assurant en quelque sorte le rôle d'annuaire du Web (Web-log), soit en des commentaires des actualités; ils prendront aussi par la suite la forme d'un journal exposant le vécu quotidien de leurs auteurs (Stassin 2016: 25 et ss.). La dénomination de " carnet de recherche en ligne », parfois privilégiée à celle de «blog » sur Hypothèses, vise à mettre à distance cet héritage du blog comme espace d'écriture de l'intime pour en asseoir la légitimité scientifique.

Dans ce contexte, la construction d'un ethos de chercheur fiable est d'autant plus importante que l'autopublication d'écrits scientifiques est entachée d'un soupçon d'illégitimité, ceux-ci ne faisant pas l'objet d'une révision préalable par les pairs. Que devient alors la construction discursive de l'ethos dans cet environnement? 
L'interdiscours mobilisé au sein des billets de blogs étudiés, on l'a vu, déborde désormais les énoncés des positions concurrentes en vigueur dans le champ disciplinaire des auteurs. De ce fait, le chercheur se positionne à présent non seulement comme scientifique rigoureux, mais aussi comme partie prenante de la société : cette double composante se manifeste à travers les billets du corpus.

Sur UC@ctualités (corpus A), qui inscrit le discours scientifique dans l'après des attentats ayant marqué l'année 2015, chaque auteur se positionne comme chercheur qui, participant tout d'abord de l'émotion ressentie par la communauté citoyenne, appréhende progressivement les événements avec la rigueur de sa profession. L'ethos se marque ainsi d'un point de vue langagier par le recours à des pronoms de première personne qui rendent compte de ce glissement : que ce soit un «nous » dont le référent bascule progressivement de la communauté citoyenne à la communauté de chercheurs (billet 2), un «je » enregistrant dans un premier temps la réaction «sur le vif» du citoyen pour ensuite prendre en charge l'énonciation du chercheur en littérature (billet 4) ou à nouveau de ce «nous » citoyen qui sollicite les éclaircissements d'un chercheur interviewé (billet 6). Le billet 3, seul billet du corpus témoignant de la désinscription de l'auteur, met en scène un historien soucieux de poser des questions, de formuler une problématique claire, de qualifier adéquatement les événements et de les inscrire dans une historicité, en documentant les termes "terreur", "attentats » ainsi que leurs évolutions.

Cette tendance est également présente au sein du second corpus où les locuteurs construisent, par des biais différents toutefois, cet ethos de chercheur-citoyen. Les auteurs de ParenThèses se donnent à voir en historiens soucieux de suivre les règles de scientificité en vigueur dans leur discipline par l'usage de sources authentifiées, d'une méthode critique, le refus des anachronismes ou de toute instrumentalisation politique des faits historiques. La concurrence d'historiens non certifiés (« il ne s'agit pas d'une profession protégée » [corpus $\mathrm{B}$, billet 2]) est bien présente dans les médias et nuit à l'image de la recherche: ils cherchent à s'en distancier. À cet égard, les figures de Stéphane Bern et de Lorànt Deutsch sont régulièrement convoquées comme repoussoir, en tant qu'historiens amateurs souvent sollicités au détriment d'historiens de profession. Au sein des billets, les auteurs veillent à expliciter leur méthode de travail et référencer les archives, y renvoyant par hyperliens cliquables le cas échéant (billets 2 et 4). Ils se montrent préoccupés des questions de validité des sources, du positionnement intellectuel dans un champ de recherche (ainsi qu'en témoigne l'interview d'un chercheur réalisé dans le billet 3). Enfin, l'un des billets - qui consiste en la relation de la soutenance de thèse de l'un des deux bloggeurs - s'interroge non sans malice sur la place qu'occupe ce genre de discours au sein de la discipline, dans le souci de ne pas délégitimer le contenu du carnet de recherche. Le locuteur tranche: "On n'a qu'à dire qu'il s'agit de la retranscription de notes ethnographiques » - et titre donc le billet « Observation (pas trop) participante d'une défense de thèse » (billet 6).

Mais la construction de cet ethos d'historien rigoureux se double d'une volonté délibérée de casser l'image de l'universitaire sérieux enfermé dans sa tour d'ivoire et ce, à deux niveaux. D'une part, on l'a déjà signalé, l'historien se montre concerné par les possibilités d'utilisation de l'histoire à des fins politiques, contre laquelle il souhaite prévenir l'allocutaire qui n'est pas forcément envisagé comme un universitaire. On trouve un exemple manifeste de cette entreprise dans le billet 5 , qui consiste en une recension très critique (c'est le moins que l'on puisse dire) d'un ouvrage de Casali, $L a$ 
longue montée de l'ignorance. L'énonciation, très engagée, s'écarte de toute idée de neutralité axiologique ("Écoeuré, je suis écoeuré »; «j'ai l'impression de m'être fait carjacker intellectuellement »; " un potentiel élevé de nuisance intellectuelle», etc.). Toutefois, le citoyen-chercheur se montre aussi comme un universitaire attaché aux valeurs éthiques de sa profession, qui prend la peine de situer Casali dans un horizon scientifique, culturel et médiatique, de citer des extraits de l'ouvrage auxquels il réagit, de pointer les lacunes bibliographiques, et de faire porter les critiques sur des éléments d'ordre épistémique («Quels indicateurs? On ne le saura jamais ») - suivant en cela le format attendu de la recension d'ouvrage.

D'autre part, ce carnet de recherche se démarque par une touche humoristique omniprésente, qui agrémente la lecture des billets. L'usage de l'humour est questionné de manière réflexive dans un billet hyperlié au billet $6^{7}$. Les auteurs exposent à la suite de Bartlett (2014) en quoi l'humour manié à bon escient faciliterait l'appropriation des savoirs: il n'y aurait donc pas lieu de s'en priver, a fortiori dans les espaces de publications comme Hypothèses qui autorisent des formes plus légères et moins conventionnelles de communication. Dans ParenThèses, l'humour se manifeste ainsi par le recours massif aux figures de l'ironie et de l'allusion, qui reposent sur un implicite partagé par l'auditoire ; il est également construit par des éléments visuels (gifs animés, ou illustrations du billet 5, p. ex.) qui entrent en résonance avec les éléments discursifs. Par ailleurs, un certain nombre d'énoncés d'archive convoqués - la plupart du temps par le biais d'hyperliens cliquables menant vers des extraits YouTube (cf. infra), mais

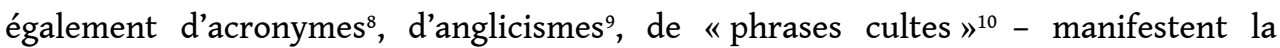
connaissance d'une culture populaire dont la reprise et la mise en relation avec les énoncés de l'archive scientifique contribuent à nourrir l'effacement des frontières déjà signalé.

On voit ainsi que le chercheur-locuteur des billets étudiés, dans le but d'asseoir sa légitimité, se présente à la fois comme respectueux des cadres et méthodes de sa discipline, mais également comme un citoyen intégré dans une sphère d'activités plus large ou, si l'on suit la terminologie de Maingueneau pour en revenir au plan du discours, actif sur une scène d'énonciation à la fois scientifique et extra-scientifique (2009 [1998]). Le savoir-faire du chercheur ne se marque pas ici par l'effacement énonciatif et l'appropriation d'un habitus discursif, mais par la mise en scène d'une méthodologie rigoureuse conformément à un ethos caractéristique du discours scientifique, qui entend servir à produire des connaissances utiles à des nonuniversitaires, sans qu'il s'agisse pour autant de vulgarisation.

\section{3. À travers les discours, quelle(s) communauté(s) ?}

L'humour déployé dans le carnet ParenThèses repose, outre sa composante visuelle et technique, sur l'ironie et l'allusion, figures supposant un implicite source de connivence entre le locuteur et son auditoire. Pour être efficaces, ironie et allusion nécessitent d'être adressées à une communauté partageant un même bagage culturel et informationnel, ainsi que des références communes. De ce fait, Perelman et OlbrechtsTyteca, qui soulignent le rôle argumentatif des figures, qualifient l'allusion de « figure de la communion » par laquelle «l'on s'efforce de créer ou de confirmer la communion avec l'auditoire» (Traité 1988 [1958]: 239 et ss.). L'apostrophe, autre figure de la communion identifiée par les auteurs du Traité, est très présente dans les billets de ParenThèses, témoignant d'un lien fort noué au lectorat anticipé (par exemple : «vous 
verrez, il y aura de quoi discuter » [Corpus B, billet 4]; "Avouez! Vous pensez à la même chose que moi " [Corpus B, billet 5]). Les apostrophes au lecteur sont en revanche marginales dans les textes d'UC@ctualité (on relève une question rhétorique, « est-ce mieux? ", dans le billet 4 du corpus A).

Enfin, les billets recourent d'une manière particulière à la citation, autre figure de la communion signalée par Perelman et Olbrechts-Tyteca qui renseigne d'ailleurs sur l'interdiscours mobilisé. On sait la place prépondérante qu'occupent les sources théoriques dans les textes scientifiques : ceux-ci s'appuient en effet sur une production de savoir antérieure par rapport à laquelle se situe toute connaissance nouvelle produite dans une activité de recherche. Or, la citation d'écrits scientifiques reste assez marginale au sein des billets étudiés dans ParenThèses, à l'exception du compte rendu, qui assez logiquement cite des extraits de l'ouvrage recensé (corpus B, billet 5). Les citations émanent par contre de sources primaires (pièces d'archives : corpus B, billets 2 et 4) - auxquels s'ajoutent les nombreuses citations issues de la culture populaire disséminées dans l'ensemble des billets, qui peuvent également prendre la forme de gifs animés ou d'hyperliens renvoyant vers un extrait vidéo diffusé sur YouTube. On trouve par contre davantage de références à la littérature scientifique sur UC@ctualité, dans le billet «Que faire? » (corpus A, billet 2), ainsi que dans « Les attentats... et après quoi ?» (corpus A, billet 3), tandis qu'« Une sociologie des attentats» (corpus A, billet 6) consiste en l'interview d'un chercheur suite à la parution de son livre.

Le carnet UC@ctualité, par ailleurs, ne recourt pas aux procédés humoristiques - sans doute le référent ne s'y prête-t-il pas. En revanche, on peut y observer l'usage d'une figure comme l'hypotypose, catégorisée par Perelman et Olbrechts-Tyteca parmi les «figures de la présence » qui ont pour effet de « rendre présent à la conscience l'objet du discours» (1988: 235). Ainsi, l'évocation d'une expérience humaine susceptible de faire écho chez le lecteur : " Il y a ainsi des textes, des phrases, des tableaux que l'on intériorise et qui, sommeillant en vous, viennent à se réveiller à l'occasion d'un événement comme pour y porter un peu de leur pâle lumière» (corpus A, billet 4). Autre exemple, le billet Que faire? s'ouvre sur un rappel des attentats de Paris et des manifestations qui s'en sont suivies:

Le Grand Duduche, le Roi des Cons mais aussi les Pandas (dans la Brume) et « celui qui n'aimait pas les gens " (enfin, certains), entre autres, ont été assassinés un mercredi matin de janvier 2015. Des Lumières se sont éteintes que de nombreuses bougies ont tentées de raviver quelques jours plus tard sur une place devenue «Chose Publique ». Espace public? Tout le monde, tout le Monde ou presque, était «Charlie ». Paris redevenait porteur d'une valeur culturelle universelle : «Citoyen, quel est ton nom? » (corpus A, billet 2).

31 Selon les propres termes de l'auteur, il s'agit là de l'« écriture automatique de motssymboles, porteurs d'images pour le monde " (ibid.) susceptibles de réactiver, chez le lecteur, l'émotion ressentie à la suite des attentats contre Charlie Hebdo. Le rappel d'un vécu commun aux Français est ici utilisé pour questionner la distance par rapport à d'autres attentats ayant émaillés l'année 2015, à d'autres endroits du monde, sans pour autant provoquer une mobilisation semblable à celle du mouvement rassemblé sous la bannière «Je suis Charlie ». Le billet lance opportunément une réflexion sur le rôle du discours comme lien social, et sur la fonction des chercheurs en informationcommunication dans le décryptage de la communication médiatique des idéologies structurant les communautés. Les éléments iconographiques co-construisent également l'énonciation de ce billet: par exemple, l'image d'en-tête, représentant le 
passe-muraille de Montmartre ${ }^{11}$, fait écho à l'évocation des frontières invisibles qui font barrière au partage de l'émotion.

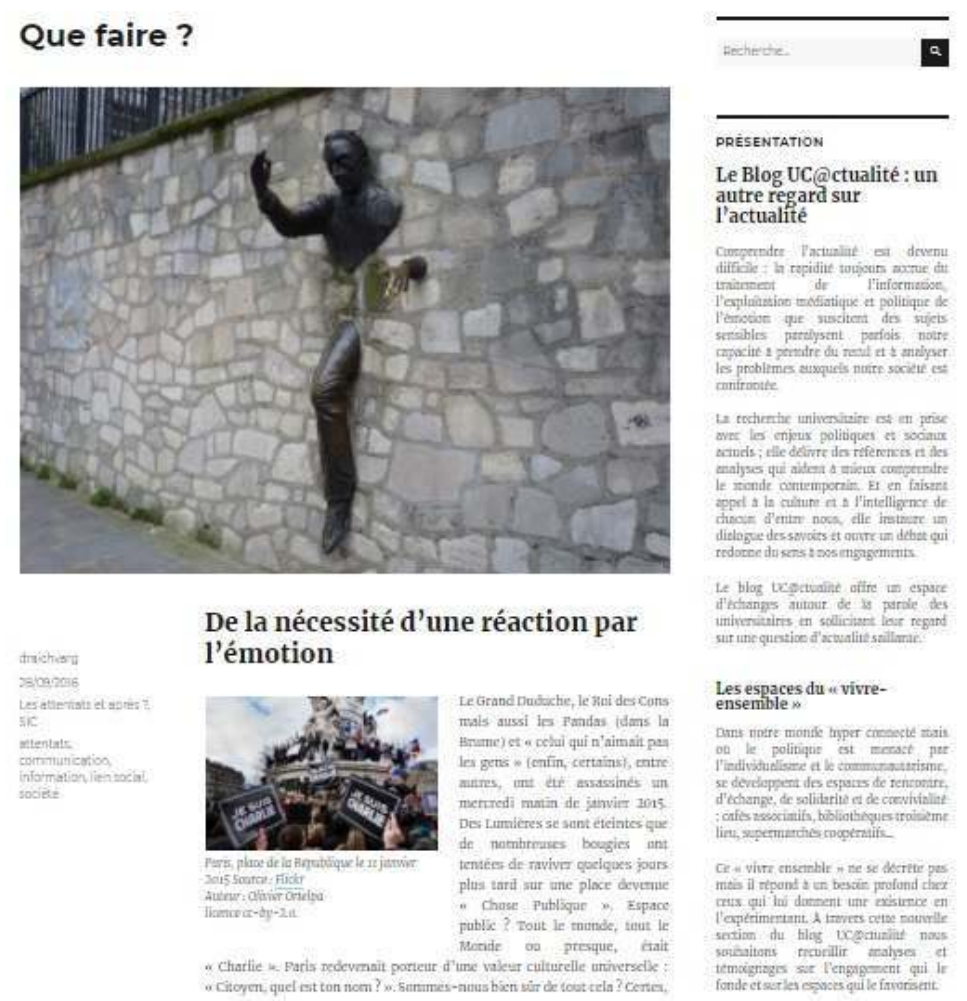

Fig. 3 : Bandeau du billet « Que faire » (corpus A, billet 2) (capturé le 23 novembre 2017)

Figures de la communion et de la présence sont liées, dans la tradition rhétorique, au genre épidictique ; un genre non pas à visée mais à dimension argumentative, reposant sur le pathos qui entretient chez le citoyen une disposition à l'action. Bien entendu, les textes scientifiques abordés ici ne peuvent être assimilés à des discours épidictiques. Mais la dimension argumentative de ces textes semble concerner le citoyen au moins autant que le scientifique. Quelle est alors l'action visée sur l'auditoire par ce recours au pathos? On peut sans doute y discerner le souci de convaincre l'auditoire du bienfondé d'une approche des questions sociales par les humanités, et une volonté de situer cette recherche dans un cadre global. Les figures intègrent le lecteur, universitaire ou non, dans une communauté sociale qui excède le champ académique; le recours à l'émotion - on l'a vu dans le cas de l'humour, c'est également le cas pour le recueillement - est de nature à favoriser l'appropriation des savoirs en vue d'une utilisation ultérieure par l'action citoyenne.

\subsection{Technologies discursives}

Les deux carnets étudiés recourent à divers degrés aux procédés technodiscursifs, associant les qualités langagières et techniques du discours numérique. Dans les limites de cette contribution, je m'attacherai uniquement à poursuivre la réflexion sur les figures rhétoriques; on gardera cependant à l'esprit que, dans l'environnement numérique, l'allocutaire est invité à prendre part à l'écriture du texte en effectuant des choix d'affichage et de paramétrage, ainsi que des choix de navigation dans le blog par l'activation des filtres de catégories ou de mots-clés, voire de mots cliquables ou 
technomots (Paveau 2012) figurant au sein des billets qui dessinent un parcours de lecture à travers l'archive. À cet égard, ParenThèses se démarque comme reposant sur une intrication étroite des billets, soulignant la continuité de la réflexion dans le temps long et les échanges entre ses deux animateurs ${ }^{12}$; à laquelle s'ajoutent de nombreux renvois vers des pages externes.

Certaines figures observées dans le corpus ne peuvent advenir, se matérialiser, sans action concrète de l'allocutaire : reposant sur des technomots, elles nécessitent en effet l'activation d'un signe-passeur (Davallon et al. 2003) et la délinéarisation du discours qui en découle pour exister. L'usager participe pleinement de la construction du sens à travers cette opération (Paveau 2016 ; Saemmer 2015). On peut citer, à titre d'exemple, cet extrait du billet 3 (corpus B) :

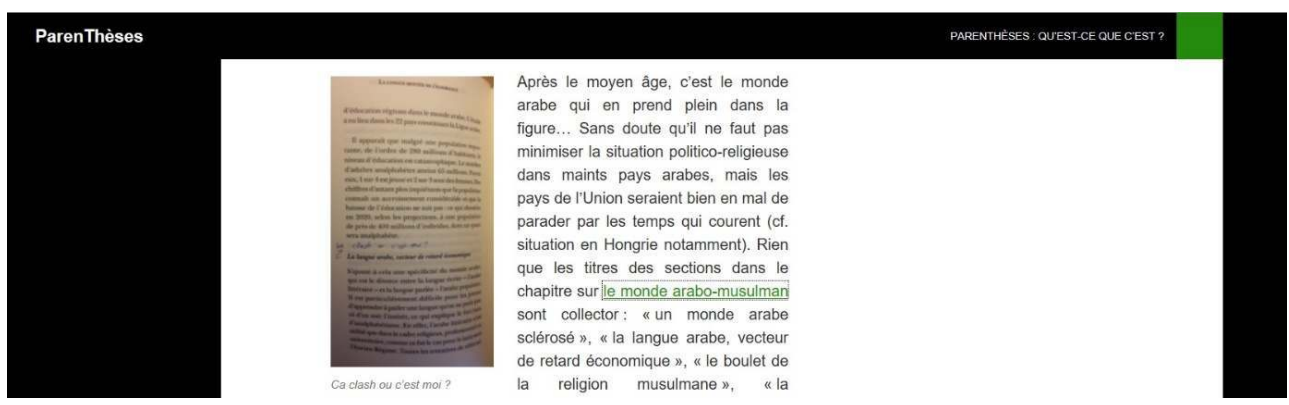

Fig. 4 : extrait du billet 3, corpus B (capturé le 14 novembre 2017)

Aucune allusion ou citation au sens classique du terme, mis à part les technomots «le monde arabo-musulman» [soulignés, et en contexte signalés par une couleur particulière] renvoyant à un extrait du film OSS 117: Le Caire, nid d'espions ${ }^{13}$ où le protagoniste principal fait montre d'une totale ignorance sur la question. Seule l'activation du lien par l'écrilecteur, toutefois, permet de saisir l'allusion amenée par la citation de l'extrait.

Les pratiques de citation des sources font également appel aux textes hyperliés, permettant éventuellement au lecteur de les vérifier par lui-même ainsi que l'avait relevé Chartier (voir plus haut). Certains billets du corpus fournissent l'accès direct aux références citées: c'est par exemple le cas dans le billet 2 du corpus $B$, où de nombreuses sources primaires (essentiellement des discours politiques) peuvent être consultées sur des sites externes par l'activation de technomots. Cette pratique de renvoi vers les sources existe également sur UC@ctualité, par exemple dans le billet 3 où, en bibliographie, la Revue blanche est liée à la page Wikipédia présentant le périodique, et où des livres cités figurent en vignettes cliquables sur le côté gauche du billet :

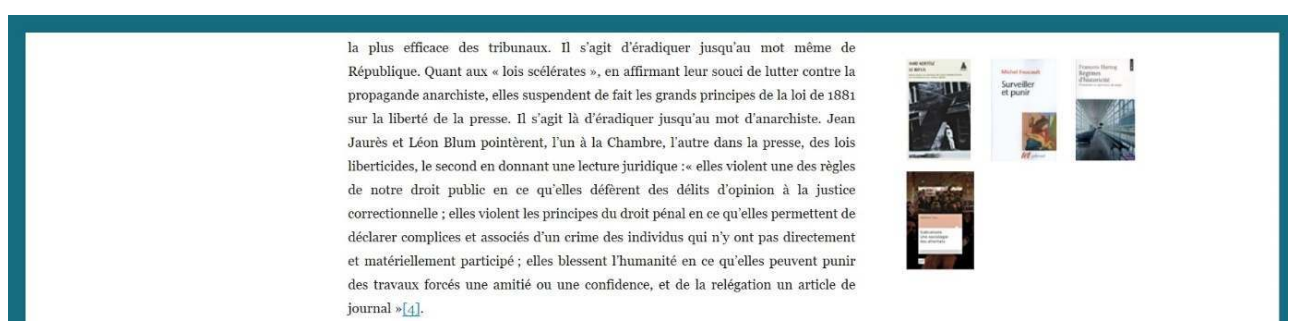

Fig. 5 : extrait du billet 3, corpus A (capturé le 14 novembre 2017) 
Les éléments technodiscursifs participent ainsi d'une sollicitation directe de l'allocutaire qui se voit dès lors invité à prendre une part active dans le processus d'appropriation des savoirs.

\section{Conclusion}

38 Je voudrais à présent tirer quelques éléments de conclusion, dans les limites permises par une analyse forcément restreinte sur un corpus qui l'est tout autant.

Premièrement, le discours scientifique produit en régime d'Open Science ne peut plus être considéré comme un discours fermé, même si ce sont avant tout les caractéristiques techniques des modes de diffusion de la science ouverte qui offrent la possibilité que ces écrits soient lus par un public extra-académique. Les discours d'escorte (des pouvoirs politiques comme l'UE, ou au niveau de la plateforme Hypothèses ) encouragent de fait les chercheurs à tenir compte de ce nouveau public; et certains d'entre eux - c'est le cas dans les billets étudiés - ajustent leurs pratiques à cette injonction. C'est à ce niveau que le concept de dimension argumentative prend son utilité : ce ne sont pas seulement les technologies discursives des écrits scientifiques en ligne qui provoquent la transformation du discours scientifique, même s'ils sont essentiels, mais bien la prise en compte d'un public plus large qu'autorisent les modes de diffusion de la recherche dans l'environnement numérique.

De ce fait, la finalité en vue de laquelle s'exerce la dimension argumentative des textes scientifiques peut se trouver modifiée. La dimension argumentative des discours étudiés ici porte non tant sur une prise de position dans un champ scientifique défini que sur l'utilité sociale de savoirs et méthodes en Humanités, dont il s'agit de convaincre l'allocutaire. Si la prise de position à l'encontre de pairs existe dans ParenThèses (ex. corpus B, billets 3 et 5), il s'agit davantage de proposer une image de la recherche en train de se faire suivant les règles de l'art, potentiellement utile à un public profane qui au demeurant goûtera les allusions à la culture populaire. Ce positionnement se trouve également sur UC@ctualité (ex. billet 2, corpus A), qui sert de façon similaire une finalité externe à la progression d'un champ de recherche puisqu'il s'agit de fournir une grille d'analyse à des faits d'actualité. Dans l'un et l'autre cas, l'interdiscours mobilisé inscrit la recherche dans un contexte social plus large qui dépasse le champ académique. Par ailleurs, les marques discursives qui construisent ces deux types d'actualité, sociale et professionnelle (par exemple les apostrophes à l'allocutaire qui ont été signalées) lient l'énoncé à sa situation d'énonciation et placent le discours scientifique sur un plan embrayé, alors qu'il est ordinairement réputé ne pas l'être (Maingueneau 2009 [1998] : 91). On signalera toutefois une certaine continuité des usages en ce sens qu'il s'agit toujours bien, à l'instar de ce qui se fait dans les publications scientifiques imprimées, de présenter la figure d'un locuteur-chercheur à même de servir de caution à la validité des savoirs diffusés ${ }^{14}$.

41 Ensuite, les stratégies rhétoriques d'adaptation à ce public anticipé sont de nature à favoriser l'appropriation des connaissances de manières variées. On peut ici les regrouper en trois catégories: (1) la construction d'une autorité discursive visant à légitimer la démarche du chercheur en Humanités et à justifier la scientificité des écrits de blogs ${ }^{15}$, (2) le recours aux émotions que suscite le pathos des figures amenant le rire, 
le recueillement ou le sentiment de partage, ainsi que (3) l'usage des technologies discursives engageant le lecteur à vérifier des sources et se documenter.

Enfin, on pourrait s'interroger sur la tension entre l'hétérogénéité du public que rend possible le brouillage des frontières de la communication scientifique sur blog, et l'abondance des figures de la présence et de la communion qui visent à renforcer le sentiment d'inclusion dans une communauté1 ${ }^{16}$. Sans trancher nécessairement ici la question, cette tension pourrait se résoudre dans la dimension d'une science citoyenne, souvent liée à l'Open Science; soit une science au service de l'intérêt général, qui voit le citoyen prendre part à la recherche mais également le chercheur agir en tant que citoyen (Stilgoe 2009). Cette tension manifeste, une fois de plus, la volonté d'inscrire la recherche dans un contexte social plus large, comme activité citoyenne à part entière.

La notion de dimension argumentative se révèle opératoire pour traiter des écrits scientifiques en accès ouvert, dans les limites du corpus étudié du moins. Le concept reste toutefois très en prise sur une conception classique de l'analyse du discours portant sur des corpus oraux et imprimés, en ce qu'il ne tient pas compte de l'énonciation éditoriale ou visuelle des écrits d'écran, par laquelle se construit d'une part une image de l'allocutaire par l'inscription dans un univers culturel donné et d'autre part une autorité énonciative à travers les logiques de réquisition qui poussent l'allocutaire à agir sur le texte. On pourrait par ailleurs s'interroger de manière plus approfondie sur le statut des ressources hyperliées (vidéos YouTube, articles, etc.), qu'on pourrait rapprocher des moyens de preuve extra-techniques ${ }^{17}$ - non construites discursivement par l'orateur - de la rhétorique classique. Une telle approche pourrait s'appuyer sur les travaux déjà mentionnés (Saemmer 2015 ; Paveau 2016).

\section{BIBLIOGRAPHIE}

Tous les liens de l'article ont été vérifiés le 22 juin 2017.

\section{Sources primaires}

Corpus A

Caron, Jean-Claude. 2016. « Les attentats... et après quoi ?». UC@ctualité. Publié le 28 septembre 2016. URL : https://ucactualite.hypotheses.org/63. [billet 3]

Mesnard, Philippe. 2016. «L'imagination de la terreur». UC@ctualité. Publié le 28 septembre 2016. URL : https://ucactualite.hypotheses.org/82 [billet 4]

Meyer, Camille. 2016. « Une sociologie des attentats ». UC@ctualité. Publié le 9 décembre 2016. URL : https://ucactualite.hypotheses.org/173 [billet 6]

Pôle éditorial numérique [Polen]. 2016. «Les dessins d'Adrien Weber, quelques jours après les attentats du 13 novembre ».UC@ctualité. Publié le 6 octobre 2016. URL : https:// ucactualite.hypotheses.org/109 [billet 5]

Raichvarg, Daniel. 2016. «Que faire ?». UC@ctualité. Publié le 28 septembre 2016. URL : https:// ucactualite.hypotheses.org/47 [billet 2] 
Zouari, Khaled, et Pascal Gay. 2016. «Les attentats, et après ? [éditorial] ». UC@ctualité. Une mise en perspective de l'actualité par les universitaires. Publié le 6 juillet 2016. URL : https:// ucactualite.hypotheses.org/category/editorial [billet 3]

Corpus B

Simon, Nicolas. 2017a. « Le moyen âge vu par les politiques ». ParenThèses. Publié le 4 avril 2017. URL : https://parenthese.hypotheses.org/1658 [billet 2]

Id. 2017b. « De Saint-Boniface à Saint-Louis : la carrière à rebondissements de Mgr. Aloïs Simon (1897-1964)». ParenThèses. Publié le 18 avril 2017. URL : https://parenthese.hypotheses.org/1689 [billet 4]

Id. 2017c. « Un livre dangereux : Dimitri Casali, 'La longue montée de l'ignorance'« . ParenThèses. Publié le 2 mai 2017. URL : https://parenthese.hypotheses.org/1728 [billet 5]

Simon, Nicolas, et Quentin Verreyden. s.d. «ParenThèses : qu'est-ce que c'est ?» ParenThèses. https://parenthese.hypotheses.org/a-propos [billet 1]

Verreycken, Quentin. 2017. « Observation (pas trop) participante d'une défense de thèse ». ParenThèses. Publié le 8 mai 2017. URL : https://parenthese.hypotheses.org/1761 [billet 6]

Verreycken, Simon [pseud. de Nicolas Simon et Quentin Verreycken]. 2017. « Droit au but : Bram De Ridder ». ParenThèses. Publié le 10 avril 2017. URL : https://parenthese.hypotheses.org/1662 [billet 3]

\section{Sources secondaires}

Amossy, Ruth. 2000. L'argumentation dans le discours (Paris : Nathan)

Amossy, Ruth. 2010. La présentation de soi : ethos et identité verbale (Paris : PUF)

Bartlett, Tom. 2014. «It's No Joke : Humor Rarely Welcome in Research Write-Ups ». The Chronicle of Higher Education, 29 septembre 2014. URL : http://www.chronicle.com/article/Its-No-JokeHumor-Rarely/149025/ ?cid =inline-promo

Beaudry, Guylaine. 2011. La communication scientifique et le numérique (Paris : Lavoisier)

Boch, Françoise \& Francis Grossmann. 2007. «L'énonciation dans les manuels scolaires de troisième ». Lidil 35 (juin, 25-40

Boch, Françoise, et Fanny Rinck. 2010. « Pour une approche énonciative de l'écrit scientifique », Lidil 41 (mai). URL : http://lidil.revues.org/3004

Bronckart, Jean-Paul, Daniel Emile Bain, Bernard Schneuwly et al. 1994 [1985]. Le fonctionnement des discours (Lausanne \& Paris : Delachaux et Niestlé)

Charaudeau, Patrick, et Dominique Maingueneau. 2002. Dictionnaire d'analyse du discours (Paris : Seuil)

Chartier, Roger. 2006. «L'écrit sur l'écran. Ordre du discours, ordre des livres et manières de lire ». Entreprises et histoire 43 (juin),15-25

Commission Européenne, éd. 2016. Open Innovation, Open Science, Open to the World: A Vision for Europe (Luxembourg : Publications Office of the European Union)

Dacos, Marin \& Pierre Mounier. 2010. « Les carnets de recherche en ligne, espace d'une conversation scientifique décentrée ». Christian Jacob éd. Lieux de savoir. 2. Gestes et supports du travail savant (Paris : Albin Michel) URL : http://archivesic.ccsd.cnrs.fr/sic_00439849/document 
Davallon, Jean, Marie Després-Lonnet, Yves Jeanneret, et al. 2003. Lire, écrire, récrire (Paris : Éd. de la Bibliothèque publique d'information. URL : http://books.openedition.org/bibpompidou/394 Jeanneret, Yves. 2014. Critique de la trivialité (Paris : Editions Non Standard) Maingueneau, Dominique. 2009 [1998]. Analyser les textes de communication (Paris : Armand Colin) Paveau, Marie-Anne. 2012. «L'intégrité des corpus natifs en ligne. Une écologie postdualiste pour la théorie du discours ». Cahiers de praxématique 59 (juin), 65-90

Paveau, Marie-Anne. 2016. « Des discours et des liens. Hypertextualite, technodiscursivite, ecrilecture ». Semen $42: 23-48$

Paveau, Marie-Anne. 2017. L'analyse du discours numérique : Dictionnaire des formes et des pratiques (Paris : Hermann)

Perelman, Chaïm \& Lucie Olbrecht-Tyteca. 1988 [1958]. La Nouvelle Rhétorique (Bruxelles : Éd. de l'Université de Bruxelles)

Rabatel, Alain. 2005. « La part de l'énonciateur dans la co-construction interactionnelle des points de vue ». Marges Linguistiques 9, 115-36

Rabatel, Alain. 2017. Pour une lecture linguistique et critique des médias (Limoges : Lambert-Lucas)

Rinck, Fanny. 2010. «L'analyse linguistique des enjeux de connaissance dans le discours scientifique ». Revue d'anthropologie des connaissances 4-3, 427-50

Saemmer, Alexandra. 2015. Rhétorique du texte numérique (Lyon : Presses de l’Enssib)

Stassin, Bérengère. 2016. La blogosphère info-doc : une communauté de savoir, une mosaïque de médiations (Toulouse : Cépaduès)

Stilgoe, Jack. 2009. Citizen Scientists. (London : Demos). URL : http://creias.ipleiria.pt/files/ 2010/08/Citizen_Scientists_-_web.pdf

Tutin, Agnès \& Francis Grossmann éds. 2013. L'écrit scientifique : du lexique au discours (Rennes : P. U. de Rennes)

Vitali Rosati, Marcello. 2016. « What is editorialization? » Sens public, janvier. URL : http:// www.sens-public.org/article1059.html

Volochinov, Valentin Nicolaïevitch. 2010. Marxisme et philosophie du langage. Éd. par Patrick Sériot (Limoges : Lambert-Lucas)

\section{NOTES}

1. L'Open Science consiste à promouvoir le libre accès aux données de la recherche ainsi qu'aux publications scientifiques, afin de favoriser la circulation des connaissances (Commission Européenne 2016).

2. Le catalogue des carnets de recherche est accessible à l'adresse https://www.openedition.org/ catalogue-notebooks. En novembre 2017, 2488 carnets y figuraient.

3. Il faudrait encore distinguer, à la suite de Boch et Grossmann (2007: §9), entre la neutralité, soit l'absence de prise de position (qui est illusoire dans le cas du discours scientifique) et l'objectivité, qui est construite par les procédés énonciatifs du discours scientifique.

4. On se référera à l'étude de Guylaine Beaudry: La communication scientifique et le numérique (2011). 
5. HYPOTHÈSES, s. d. "À propos ", Hypothèses. URL: https://fr.hypotheses.org/a-proposdhypotheses. Notons par ailleurs que la plateforme est ensuite devenue multilingue : quatorze langues sont ainsi répertoriées au catalogue (novembre 2017).

6. Il faudrait encore distinguer ici entre subjectivité déictique et subjectivité modale (Rabatel 2005) . Dans le discours scientifique traditionnel, l'effacement énonciatif concerne essentiellement la première, ce qui n'est plus le cas dans les exemples de notre corpus.

7. Quentin Verreycken, 2014. "Why so serious? De l'usage de l'humour dans la production scientifique». Billet ParenThèses, publié le 13 octobre 2014. URL: https:// parenthese.hypotheses.org/158.

8. Ex. : «Ils sont tellement YOLO en 1913 qu'ils prennent des copies authentiques d'actes du $18^{\mathrm{e}}$ siècle en séance plénière » (corpus B, billet 2). YOLO est l'acronyme de "You Only Live Once », qui peut être prononcé avant de procéder à une action dangereuse ou décalée.

9. Ex. « en termes de whathefuckesque organisationnel » (corpus B, billet 5).

10. Ex. : «Hasta la vista baby » (corpus B, billet 2) - célèbre réplique du film Terminator 2 réalisé par James Cameron en 1991.

11. Source: Flickr. Attribution: Martine. Licence (CC BY-NC 2.0)

12. Un exemple tiré du corpus $B$ : le billet 6 (relation de la défense de thèse) se clôt sur un renvoi au billet 5 (compte rendu de l'ouvrage de Casali manifestement reçu à l'issue de la soutenance de thèse en question).

13. Réalisé par Michel Hazanavicus en 2006.

14. Je remercie le relecteur de cet article d'avoir attiré mon attention sur ce point.

15. Dans le corpus, hormis les textes de présentation, un texte ne correspond clairement pas à la définition de l'écrit scientifique proposée par Rinck: il s'agit du billet « Les dessins d'Adrien Weber, quelques jours après les attentats du 13 novembre » (billet 3, corpus A). Étant donné qu'il s'agit de croquis, je n'en ai pas fait mention dans l'analyse - bien qu'il puisse être intéressant de questionner par ailleurs l'hétérogénéité des contenus dans l'économie générale d'un carnet de recherche.

16. Je remercie Grégory Cormann d'avoir attiré mon attention sur ce point.

17. Sur le rôle des textes ou documents audio-visuels, hyperliés, discours rapportés/montrés directs utilisés comme moyens de preuve, voir Rabatel (2017: 379-394).

\section{RÉSUMÉS}

La présente contribution entend questionner la dimension argumentative (Amossy 2000) d'écrits scientifiques natifs du Web que sont les billets de blog. Des travaux antérieurs ont mis en évidence le rôle majeur des moyens de persuasion qui traversent le discours scientifique. Si ce type de discours est traditionnellement considéré comme un discours fermé, à l'attention des pairs, les dispositifs numériques autorisent l'élargissement potentiel du lectorat de la recherche. L'auditoire hétérogène que présuppose le contexte de l'open Science amène dès lors à s'interroger sur les stratégies rhétoriques d'adaptation au public mises en œuvre par les carnetiers pour favoriser l'appropriation des savoirs diffusés. L'étude de la dimension argumentative des écrits de blogs, qui intègre dans l'analyse du discours scientifique la préoccupation d'une action sur l'auditoire, fournit des outils pour expliquer le recours aux moyens de preuves rhétoriques au sein de textes qui ne possèdent pas une visée argumentative explicite. Nous en testerons ici 
l'opérativité, en étudiant plus précisément deux carnets de recherche : l'un donnant la parole à des chercheurs dans le contexte de la société française post-attentats, l'autre se rattachant à l'actualité de la recherche - en l'occurrence, celle d'une thèse en cours, relatée par deux historiens.

This contribution aims to examine the argumentative dimension of scientific blog posts. Previous works have shown the important role of persuasion means in scientific discourses, usually viewed as "closed discourses" exclusively addressed to peers. However, new modes of diffusion due to the Open Science context are changing the expected audience of scientific writings and thus involve new rhetorical strategies in order to take into account a broader public. The concept of "argumentative dimension" (Amossy 2000) entails the adjustment to an audience in order to win shared acceptance of a vision and therein the use of rhetorical means of proof (ethos, logos, pathos). This article discusses this concept by way of a case study based on two academic blogs from the French platform Hypothèses: on the one hand, a blog that gives voice to French researchers after the 2015 terrorist attacks, on the other hand, a blog where two historians are relating their current $\mathrm{PhD}$ project.

INDEX

Mots-clés : blogging scientifique, dimension argumentative, discours scientifique, Open Science Keywords : academic blogging, argumentative dimension, Open Science, scientific discourse

\section{AUTEUR}

\section{INGRID MAYEUR}

Université de Liège, UR Traverses, Sémiotique et Rhétorique 